QUARTERLY OF APPLIED MATHEMATICS

VOLUME LXIX, NUMBER 4

DECEMBER 2011, PAGES 613-634

S $0033-569 X(2011) 01234-9$

Article electronically published on June 28, 2011

\title{
SHOCK WAVE FORMATION PROCESS FOR A MULTIDIMENSIONAL SCALAR CONSERVATION LAW
}

\author{
BY
}

V. G. DANILOV (Moscow Technical University of Communication and Informatics, Aviamotornaya 8a, 111024 Moscow, Russia)

AND

\section{MITROVIC (Faculty of Mathematics, University of Montenegro, Cetinjski put bb, 81000 Podgorica, Montenegro)}

Abstract. We construct a global smooth approximate solution to a multidimensional scalar conservation law describing the shock wave formation process for initial data with small variation. In order to solve the problem, we modify the method of characteristics by introducing "new characteristics", nonintersecting curves along which the (approximate) solution to the problem under study is constant. The procedure is based on the weak asymptotic method, a technique which appeared to be rather powerful for investigating nonlinear waves interactions.

1. Introduction. In the current paper, we construct an approximate (in a weak sense) solution $u_{\varepsilon}(t, x), x \in \mathbf{R}^{d}, t \in \mathbf{R}^{+}$, where $\varepsilon$ is a regularization parameter, corresponding to a multidimensional shock wave formation in the case of a Cauchy problem for a scalar conservation law.

In order to make the problem precise, assume that $\mathbf{R}^{d}$ is divided into three disjoint domains $\Omega_{L}, \Omega_{0}$ and $\Omega_{R}$, i.e., $\mathbf{R}^{d}=\Omega_{L} \dot{\cup} \bar{\Omega}_{0} \dot{\cup} \Omega_{R}$, where $\dot{U}$ denotes disjoint union. Let $\Gamma_{L}=\partial \Omega_{L}=\bar{\Omega}_{L} \cap \bar{\Omega}_{0}$ and $\Gamma_{R}=\partial \Omega_{R}=\bar{\Omega}_{R} \cap \bar{\Omega}_{0}$ (see Figure 1). Assume that $\Gamma_{L}$ and $\Gamma_{R}$ are $(d-1)$-dimensional manifolds admitting the following representation:

$$
\begin{aligned}
& \Gamma_{L}=\left\{x=\chi_{L}(s): s \in \mathbf{R}^{d-1}, \chi_{L} \in \operatorname{Lip}\left(\mathbf{R}^{d-1}\right)\right\}, \\
& \Gamma_{R}=\left\{x=\chi_{R}(s): s \in \mathbf{R}^{d-1}, \chi_{R} \in \operatorname{Lip}\left(\mathbf{R}^{d-1}\right)\right\} .
\end{aligned}
$$

Received April 24, 2009.

2000 Mathematics Subject Classification. Primary 35L65, 35L67.

Key words and phrases. global approximate solution, weak asymptotic method.

The work of V. G. Danilov is supported by RFFI grant 05-01-00912, DFG Project 436 RUS 113/895/0-1. E-mail address: danilov@miem.edu.ru

Current address: Faculty of Mathematics, University of Bergen, Johannes Bruns gate 12, 5007 Bergen.

E-mail address: matematika@t-com.me 
Now, take a Lipschitz piecewise smooth function $u_{0}: \mathbf{R}^{d} \rightarrow \mathbf{R}$ such that

$$
u_{0}(x)= \begin{cases}U_{L}, & x \in \Omega_{L}, \\ u_{1}(x), & x \in \bar{\Omega}_{0} \quad \in \operatorname{Lip}\left(\mathbf{R}^{d}\right), \\ U_{R}, & x \in \Omega_{R},\end{cases}
$$

where $u_{1} \in C^{2}\left(\Omega_{0}\right)$, and $U_{L}$ and $U_{R}$ are constants such that $\left|U_{L}-U_{R}\right|$ is small enough. We are going to consider the following problem:

$$
\begin{gathered}
\frac{\partial u}{\partial t}+\langle\nabla, f(u)\rangle=0, \\
\left.u\right|_{t=0}=u_{0}(x) \in \operatorname{Lip}\left(\mathbf{R}^{d}\right),
\end{gathered}
$$

where for $f \in C^{3}\left(\mathbf{R} ; \mathbf{R}^{d}\right),\langle\nabla, f(u)\rangle=\operatorname{div}_{x} f(u)=\sum_{i=1}^{d} \frac{\partial f(u)}{\partial x_{i}}, u=u(x, t)$. It is well known that problem (1.2), (1.3) in general does not admit a globally defined classical solution. This means that after some time the shock wave will occur, and we need to pass to the weak solution concept.
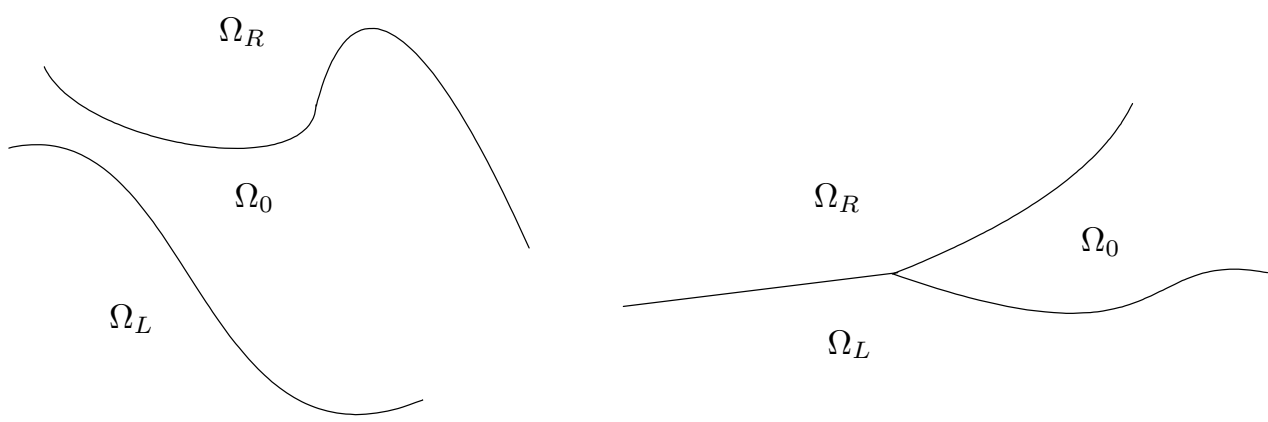

FIG. 1. The left-hand plot shows an admissible disposition of $\Omega_{0}$, $\Omega_{L}$ and $\Omega_{R}$. The right-hand profile is not admissible since the shock wave has already been formed.

Although it is well known that for any initial data $u_{0} \in L^{1}\left(\mathbf{R}^{d}\right)$ there exists a unique entropy admissible weak solution to (1.2), (1.3) (see 20]), it is very important from geometrical 19, 26, analytical [17, 18, and practical (petroleum engineering) [1, 16, 28, points of view to explicitly construct the global (approximate) solution describing the formation and propagation of the shock wave. The mentioned construction is the main contribution of the paper.

To problem (1.2), (1.3), there corresponds the following system of characteristics:

$$
\begin{aligned}
\dot{x}_{i} & =f_{i}^{\prime}(u), & \left.x_{i}\right|_{t=0} & =x_{0}, \\
\dot{u} & =0, & \left.u\right|_{t=0} & =u_{0}(x) .
\end{aligned}
$$

As is well known, problem (1.2), (1.3) will have the classical solution along the characteristics as long as the Jacobian $J=\operatorname{det} \frac{\partial x}{\partial x_{0}}$ is greater than zero. From, e.g., [26], we know that

$$
J=\operatorname{det} \frac{\partial x}{\partial x_{0}}=1+t \sum_{i=1}^{d} f^{\prime \prime}\left(u_{0}\right) \frac{\partial u_{0}}{\partial x_{0 i}} .
$$


Therefore, if we want a shock wave to appear, we must assume that $J<0$ for some $t>0$ and $x_{0} \in \mathbf{R}^{d}$. Actually, we shall assume a little bit more than the latter, that $u_{1}\left(x_{0}\right)=u_{0}\left(x_{0}\right), x_{0} \in \Omega_{0}$, satisfies the following problem:

$$
\begin{array}{r}
\sum_{i=1}^{d} f_{i}^{\prime \prime}\left(u_{1}\right) \frac{\partial u_{1}}{\partial x_{i}}=-K, \\
\left.u_{1}\right|_{\Gamma_{L}}=U_{L},\left.\quad u_{1}\right|_{\Gamma_{R}}=U_{R},
\end{array}
$$

where $K=K(s), s \in \mathbf{R}^{d-1}$, is a positive Lipschitz continuous function which is constant along the characteristics of problem (1.6). Furthermore, we shall assume that $\Omega_{0}$ admits a complete fibration along the characteristics of problem (1.6) issuing from $\Gamma_{L}$ (see Figure 2 and Remark 1.1.1).
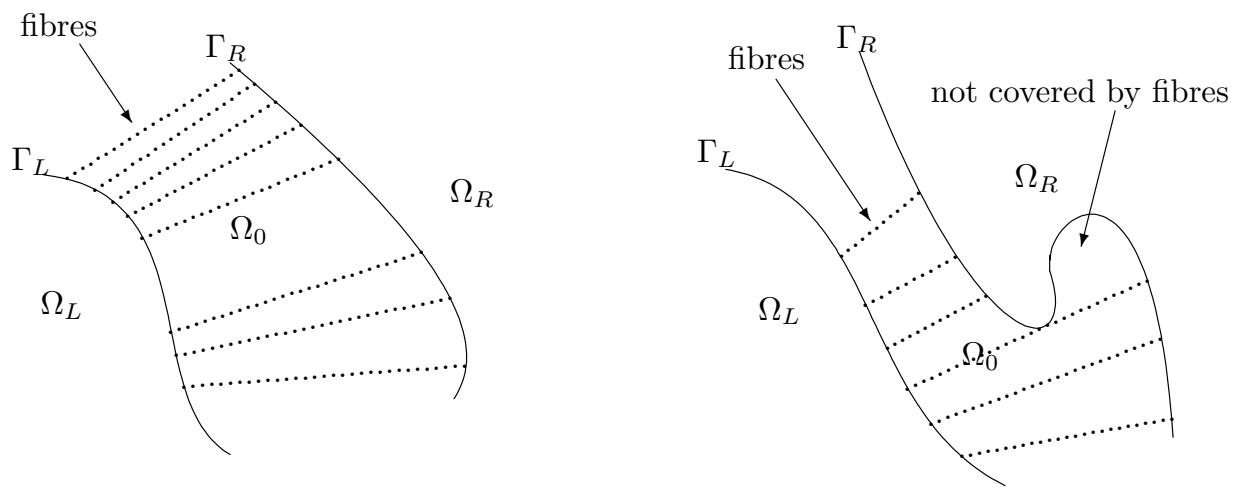

FIG. 2. On the left plot we have an admissible disposition of $\Omega_{L}, \Omega_{R}$ and $\Omega_{0}$. The situation on the right-hand side is not admissible since we have a part of $\Omega_{0}$ that is not covered by the fibres. Observe that the fibres are characteristics corresponding to (1.6), and they are not necessarily straight lines.

This is a technical assumption which provides all the characteristics issuing from the same fibre of $\Omega_{0}$ to intersect at the same point $\left(t^{*}(s), x(s)\right) \in \mathbf{R}^{+} \times \mathbf{R}^{d}$, where $s \in \mathbf{R}^{d-1}$ is the parametrization argument of $\Gamma_{L}$ (see Figure 3). It appears that this significantly simplifies the description of the shock wave formation process (see a further explanation below).

Notice that the problem of construction of a global smooth approximating solution to (1.2), (1.3) describing the formation of shock waves has already been studied by A. M. Il'in [22, 18, 23, and by the authors [7, 8, 9] in the case of one-dimensional scalar conservation laws with a convex flux $f \in C^{2}(\mathbf{R})$. In $[22$, the author considered a situation with initial data such that a classical solution blows up in a single point. His construction is based on a viscosity regularization of the considered conservation law. Using such a regularization, the author obtains a global approximating solution via a set of functional series which are defined in appropriate domains in $\mathbf{R}^{+} \times \mathbf{R}$. Then, he shows that every two such series match in domains where they are both defined. Such a method is known as the matching method. The most difficult part in the matching method was the construction 
of the approximate solution in a neighborhood of the gradient catastrophe point, i.e. the point where the classical solution blows up (see Figure 4, a)). In [7], we noticed that it is much easier to construct a global smooth approximating solution if we have a line of the "gradient catastrophe" (see Figure 4, b)). In [7, we assumed that the function $u_{1}$ from (1.1) was defined by the following implicit equation:

$$
f^{\prime}\left(u_{1}\left(x_{0}\right)\right)=-K x_{0}+b, \quad a \leq x_{0} \leq b,
$$

where $K>0$ and $b>0$ were assumed to be constants. Calculating the derivative of both sides of (1.7) we get $f^{\prime \prime}\left(u_{1}\left(x_{0}\right)\right) \frac{\partial u_{1}}{\partial x_{0}}=-K$. So, we see that (1.6) is a direct generalization of the one-dimensional situation. Actually, condition (1.7) provides all the characteristics issuing from the interval $(a, b)$ to intersect at the same point. Thus, we have the line of the gradient catastrophe (Figure $4, \mathrm{~b})$ ), and not only the point of the gradient catastrophe (Figure 4, a)).

Also, notice that problem (1.2), (2.5) was only auxiliary. In [8, 24, we considered a one-dimensional system of generalized pressureless gas dynamics. We used the explicit form of the approximate solution to (1.2), (1.3) in order to describe the formation of the delta shock wave which appears as a natural part of a solution to the considered system. In [9], we used (1.2), (1.3) (with $\left|U_{L}-U_{R}\right|$ arbitrarily small) to describe the evolution of arbitrary initial data $u_{0} \in C^{1}(\mathbf{R})$ corresponding to a one-dimensional scalar conservation law with a convex flux $f \in C^{2}(\mathbf{R})$.

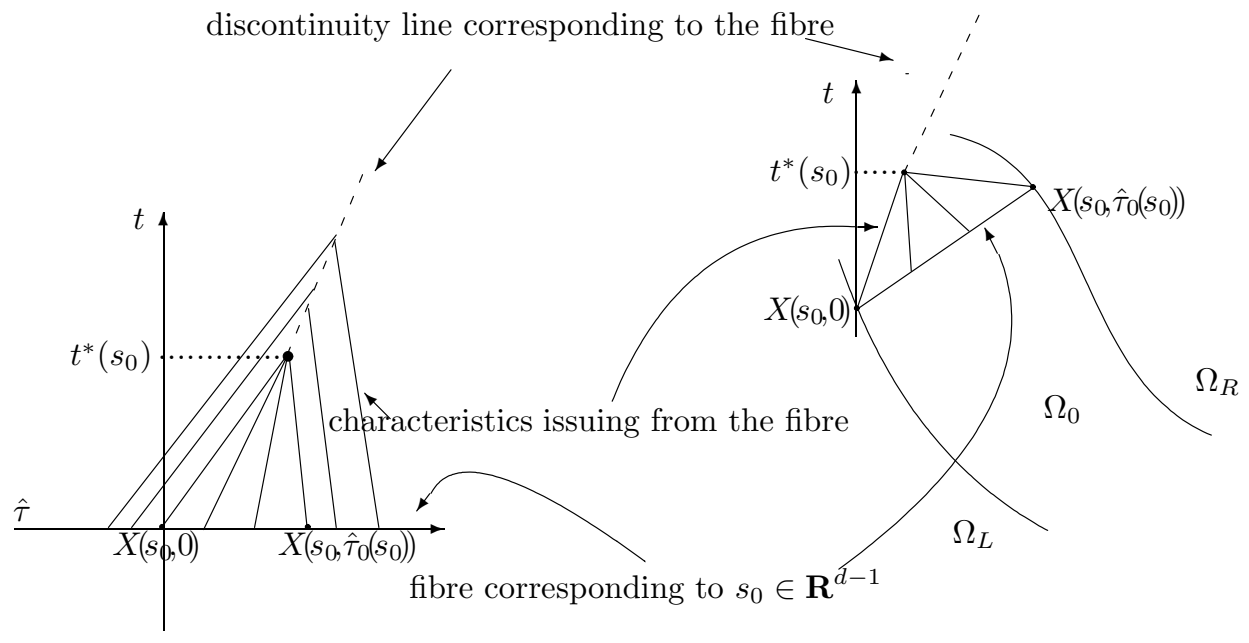

FIG. 3. Behavior of the standard characteristics issuing from the fibre corresponding to $s_{0} \in \mathbf{R}^{d-1}$ plotted in $(t, \hat{\tau}) \in \mathbf{R}^{+} \times \mathbf{R}$-plane (left plot) and in $(t, x) \in \mathbf{R}^{+} \times \mathbf{R}^{d}$-space (right plot).

In order to find the desired approximate solution of (1.2), (1.3) in the one-dimensional case, we modified the method of characteristics. More precisely, we have defined the nonintersecting curves, so-called "new characteristics", along which the approximate solution 
is constant (see Figure 6). As the regularization parameter tends to zero, the "new characteristics" tend to the Dafermos generalized characteristics [2]. Therefore, the approximate solution tends to an admissible weak solution of the considered Cauchy problem (see [7, 8]). Here, we shall present a nontrivial generalization of the latter procedure to the multidimensional case.
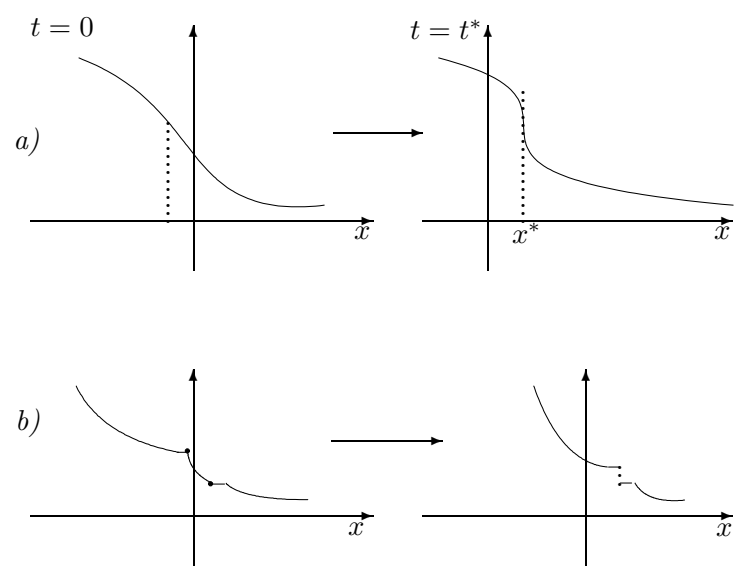

FIG. 4. Plot $a$ ) shows blowing up in a single point (the point $\left(t^{*}, x^{*}\right)$ ). Plot $b$ ) is a case when we have a line of the gradient catastrophe (the part between the dots straightens in the shock wave at the moment $\left.t=t^{*}\right)$.

Recall that in the case of initial data in the general position, at the moment of the gradient catastrophe, one point with vertical tangent arises on the graph of the solution. Moreover, small variations of arbitrary (smooth) initial data render the initial data in the general position. As one can see, we do not use the concept of construction of general position, which is standard in geometry. Actually, we replace the general position by a special one which allows us to construct an approximate solution.

REMARK 1.1. Notice that problem (1.6) is equivalent to the following system of characteristics:

$$
\begin{array}{rlrl}
\frac{d X_{i}}{d \hat{\tau}} & =f_{i}^{\prime \prime}\left(u_{1}\right), & \left.X_{i}\right|_{\hat{\tau}=0}=\chi_{L}^{i}(s), \\
\frac{d u_{1}}{d \hat{\tau}}=-K(s), & \left.u_{1}\right|_{\hat{\tau}=0}=U_{L},
\end{array}
$$

and from here we have

$$
\begin{aligned}
& u_{1}=U_{L}-K(s) \hat{\tau}, \\
& X=\int_{0}^{\hat{\tau}} f^{\prime \prime}\left(U_{L}-K(s) \tau^{\prime}\right) d \tau^{\prime}+\chi_{L}(s)=-\frac{1}{K(s)}\left(f^{\prime}\left(u_{1}\right)-f^{\prime}\left(U_{L}\right)\right)+\chi_{L}(s) .
\end{aligned}
$$

Furthermore, notice that from the boundary conditions given in (1.6), it follows that for every $s \in \mathbf{R}^{d-1}$ there exists $\hat{\tau}>0$ such that $U_{R}=U_{L}-K(s) \hat{\tau}$. Thus, we see from (1.6) that it must be

$$
U_{L}>U_{R}
$$


Notice that our assumption on the complete fibration of $\Omega_{0}$ can be written as

$$
\bar{\Omega}_{0}=\bigcup_{s \in \Gamma_{L}}\left\{X(s, \hat{\tau}): \hat{\tau} \in\left[0, \hat{\tau}_{0}(s)\right]\right\},
$$

where $X$ is given by (1.8) and $\dot{U}$ denotes the disjoint union. By $\hat{\tau}_{0}(s)$ we denote a real number such that $X\left(\hat{\tau}_{0}(s), s\right) \in \Gamma_{R}$. It is clear that $X(0, s) \in \Gamma_{L}$. Also, since $\Omega_{0}$ admits the complete fibration along the characteristics $X$, the change of coordinates $(\hat{\tau}, s) \mapsto x_{0}$, $(\hat{\tau}, s) \in \dot{\bigcup}_{s \in \mathbf{R}^{d-1}}\left[0, \hat{\tau}_{0}(s)\right] \times\{s\}$ is regular and the corresponding Jacobian $\operatorname{det}\left(\frac{\partial x_{0}}{\partial \hat{\tau}}, \frac{\partial x_{0}}{\partial s}\right)$ must be different from zero. Therefore, without losing generality, we can assume that

$$
\operatorname{det}\left(\frac{\partial x_{0}}{\partial \hat{\tau}}, \frac{\partial x_{0}}{\partial s}\right) \geq \text { const }>0, \quad(\hat{\tau}, s) \in \bigcup_{s \in \mathbf{R}^{d-1}}\left[0, \hat{\tau}_{0}(s)\right] \times\{s\} .
$$

EXAMPLE 1.2. We will give an example of problem (1.2), (1.3) satisfying the assumptions given above. A similar example is [26, Example 7.]. Consider the equation

$$
\frac{\partial u}{\partial t}+p \frac{\partial u^{2}}{\partial x_{1}}+q \frac{\partial u^{2}}{\partial x_{2}}=0
$$

where $p, q>0$. To assign initial data $u_{0} \in \operatorname{Lip}\left(\mathbf{R}^{2}\right)$, fix arbitrary constants $U_{L}>U_{R}>0$, and a positive function $K \in \operatorname{Lip}(\mathbf{R})$. Then, put

$$
\begin{aligned}
\Gamma_{L} & =\left\{\left(x_{1}, x_{2}\right) \in \mathbf{R}^{2}: x_{2}=0\right\}, \\
\Gamma_{R} & =\left\{\left(x_{1}, x_{2}\right) \in \mathbf{R}^{2}: U_{L}-K\left(x_{1}-\frac{p}{q} x_{2}\right) \frac{x_{2}}{2 q}=U_{R}, x_{2} \geq 0\right\} .
\end{aligned}
$$

Finally, we take

$$
u_{0}(x)= \begin{cases}U_{L}, & \left(x_{1}, x_{2}\right) \in \Omega_{L}, \\ U_{L}-K\left(x_{1}-\frac{p}{q} x_{2}\right) \frac{x_{2}}{2 q}, & \left(x_{1}, x_{2}\right) \in \Omega_{0}, \\ U_{R}, & \left(x_{1}, x_{2}\right) \in \Omega_{R},\end{cases}
$$

where $\Omega_{L}, \Omega_{R}$ and $\Omega_{0}$ are plotted in Figure 5. Let us prove that the function $u_{1}\left(x_{1}, x_{2}\right)=$ $U_{L}-K\left(x_{1}-\frac{p}{q} x_{2}\right) \frac{x_{2}}{2 q}$ satisfies (1.6). In our case, (1.6) reduces to

$$
\begin{gathered}
2 p \frac{\partial u_{1}}{\partial x_{1}}+2 q \frac{\partial u_{1}}{\partial x_{2}}=-K\left(x_{1}\right), \\
\left.u_{1}\right|_{\Gamma_{L}}=U_{L},\left.\quad u_{1}\right|_{\Gamma_{R}}=U_{R} .
\end{gathered}
$$

The corresponding system of characteristics is

$$
\left\{\begin{array}{l}
\dot{x}_{1}=2 p, \quad \dot{x}_{2}=2 q, \quad \dot{u}_{1}=-K\left(x_{10}\right), \\
x_{1}(0)=x_{10}, \quad x_{2}(0)=0, \quad u_{1}(0)=U_{L},
\end{array}\right.
$$

whose solution is

$$
\begin{gathered}
x_{1}=2 p \hat{\tau}+x_{10}, \quad x_{2}=2 q \hat{\tau}, \\
u_{1}=U_{L}-K\left(x_{10}\right) \hat{\tau}, \quad \hat{\tau}>0,
\end{gathered}
$$

and from this system, we finally conclude $u_{1}\left(x_{1}, x_{2}\right)=U_{L}-K\left(x_{1}-\frac{p}{q} x_{2}\right) \frac{x_{2}}{2 q}$, which is exactly the form of the function $u_{0}$ on $\Omega_{0}$. 


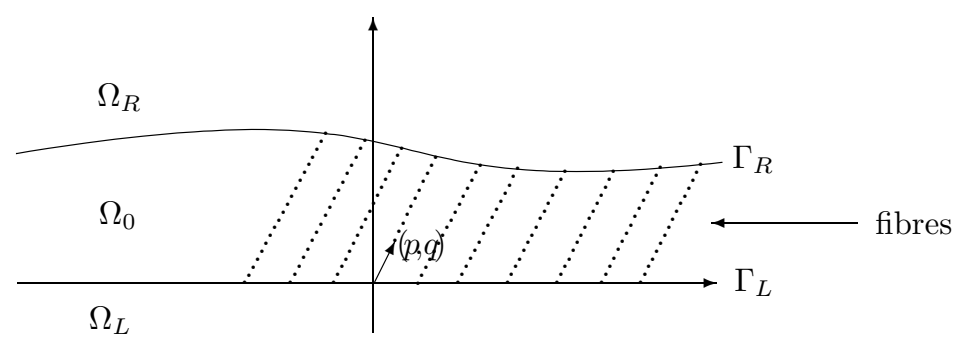

FIG. 5. If 1.13 models a traffic flow, then the vector $(p, q)$ determines the direction of the traffic while the unknown function $u$ represents the density of the vehicles.

For instance, if we take $\Gamma_{L}$ as above and $\Gamma_{R}=\{(x, 2): x \in(-\infty,-1]\} \cup\left\{\left(x, x^{2}+1\right)\right.$ : $x \in(-1,0]\} \cup\{(x, 1): x \in(0, \infty)\}, p=1, q=1 / 2$, then we must have

$$
U_{R}=U_{L}-K\left(x-\frac{1}{2}\left(x^{2}+1\right)\right) \frac{x^{2}+1}{4} \Longrightarrow K(y)= \begin{cases}\frac{4\left(U_{L}-U_{R}\right)}{(1+\sqrt{2})^{2}+1}, & y \leq-2, \\ \frac{4\left(U_{L}-U_{R}\right)}{(1+\sqrt{-y})^{2}+1}, & -2<y<-\frac{1}{2}, \\ \frac{4\left(U_{L}-U_{R}\right)}{(1+\sqrt{1 / 2})^{2}+1}, & y \geq-\frac{1}{2} .\end{cases}
$$

The paper is organized as follows.

In Section 2, we solve an auxiliary Cauchy problem whose weak asymptotic solution actually represents the weak asymptotic solution to (1.2), (1.3). The latter fact is proved in Section 3.

2. Approximate equation. In this section, we shall introduce and solve a family of problems whose solutions will represent the wanted approximating solution to (1.2), (1.3). In the beginning, we introduce definitions and the fundamental theorem of the method that we are going to use: the weak asymptotic method. The family of approximating solutions constructed in such a way will be called the weak asymptotic solution. The method is intensively used in recent years for investigations of nonlinear wave phenomena. For instance, using this method, we are able to find explicit formulas describing the interaction of solitons in the case of generalized KdV equations [5, 10, the interaction of Sine-Gordon solitons [14, 21, the evolution of nonlinear waves in the case of scalar conservation laws [3, 7], the interaction [6, 11] and formation [8, 25] of $\delta$-shock waves in the case of a triangular system of conservation laws, $\delta^{\prime}$-shock waves as a new type of singular solution of hyperbolic systems of conservation laws [27, the confluence of free boundaries in the Stefan problem with underheating [4, and different interactions of the shock waves appearing on the gas dynamics [12, 13, 15, etc.

In the sequel, we imply that $t \in \mathbf{R}^{+}$and $x \in \mathbf{R}^{d}$. Also, for a differentiable function $f: \mathbf{R}^{d} \rightarrow \mathbf{R}$, we write

$$
\nabla f=\nabla_{x} f=\left(\frac{\partial f}{\partial x_{1}}, \ldots, \frac{\partial f}{\partial x_{d}}\right)
$$


If $s=\left(s_{1}, \ldots, s_{m}\right): \mathbf{R}^{d} \rightarrow \mathbf{R}^{m}$ is a differentiable function depending on $x \in \mathbf{R}^{d}$, then we denote

$$
\frac{\partial s}{\partial x}=\left(\begin{array}{cc}
\frac{\partial s_{1}}{\partial x_{1}} \cdots & \frac{\partial s_{1}}{\partial x_{d}} \\
\vdots & \vdots \\
\frac{\partial s_{m}}{\partial x_{1}} \cdots & \frac{\partial s_{m}}{\partial x_{d}}
\end{array}\right) \in M^{m \times d},
$$

where $M^{m \times d}$ is the space of the matrix with $m$ rows and $d$ columns.

By $\operatorname{det}(a, b)$ we denote the determinant of the matrix $(a, b) \in M^{d \times d}$, where $a \in M^{d \times k}$ and $b \in M^{d \times(d-k)}$.

For two vectors $x, y \in \mathbf{R}^{d}$, we write

$$
\langle x, y\rangle=\sum_{i=1}^{d} x_{i} y_{i} \text { and } x y=\left[x_{i} y_{j}\right]_{i, j=1, \ldots, d} \in M^{d \times d} .
$$

Definition 2.1. By $O_{\mathcal{D}^{\prime}}\left(\varepsilon^{\alpha}\right) \subset \mathcal{D}^{\prime}\left(\mathbf{R}^{d}\right), \alpha \in \mathbf{R}$, we denote the family of distributions depending on $\varepsilon \in(0,1)$ and $t \in \mathbf{R}^{+}$such that for any test function $\eta(x) \in C_{0}^{1}\left(\mathbf{R}^{d}\right)$, the estimate

$$
\left\langle O_{\mathcal{D}^{\prime}}\left(\varepsilon^{\alpha}\right), \eta(x)\right\rangle=O\left(\varepsilon^{\alpha}\right), \quad \varepsilon \rightarrow 0,
$$

holds, where the term on the right-hand side is understood in the usual Landau sense and locally uniformly in $t$, i.e., $\left|O\left(\varepsilon^{\alpha}\right)\right| \leq C_{T} \varepsilon^{\alpha}$ for $t \in[0, T]$, where $C_{T}$ is a constant depending only on $T$.

By $o_{\mathcal{D}^{\prime}}(1) \subset \mathcal{D}^{\prime}\left(\mathbf{R}^{d}\right)$ we denote a family of distributions depending on $\varepsilon \in(0,1)$ and $t \in \mathbf{R}^{+}$such that for any test function $\eta(x) \in C_{0}^{1}\left(\mathbf{R}^{d}\right)$, the estimate

$$
\left\langle o_{\mathcal{D}^{\prime}}(1), \eta(x)\right\rangle=o(1), \quad \varepsilon \rightarrow 0,
$$

holds, where the estimate on the right-hand side is understood in the usual Landau sense and locally uniformly in $t$, i.e., $|o(1)| \leq C_{T} g(\varepsilon)$ for $t \in[0, T]$, where $g$ is a function tending to zero as $\varepsilon \rightarrow 0$, and $C_{T}$ is a constant depending only on $T$.

Definition 2.2. The family of functions $\left(u_{\varepsilon}\right)=\left(u_{\varepsilon}(t, x)\right) \subset C^{1}\left(\mathbf{R}^{+} ; \mathcal{D}^{\prime}\left(\mathbf{R}^{d}\right)\right)$ is called a weak asymptotic solution of problem (1.2), (1.3) if

$$
\begin{aligned}
& \frac{\partial u_{\varepsilon}}{\partial t}+\left\langle\nabla, f\left(u_{\varepsilon}\right)\right\rangle=o_{\mathcal{D}^{\prime}}(1), \\
& \left.u_{\varepsilon}\right|_{t=0}-u_{0}=o_{\mathcal{D}^{\prime}}(1), \quad \varepsilon \rightarrow 0 .
\end{aligned}
$$

Observe that a sequence of solutions obtained by the standard vanishing viscosity approximation of (1.2) is a special case of the weak asymptotic solution. Indeed, in the framework of the vanishing viscosity approach, we consider the following sequence of equations:

$$
\frac{\partial u_{\varepsilon}}{\partial t}+\left\langle\nabla, f\left(u_{\varepsilon}\right)\right\rangle=\varepsilon \partial_{x x} u_{\varepsilon}
$$

and, clearly, $\varepsilon \partial_{x x} u_{\varepsilon}=o_{\mathcal{D}^{\prime}}(1)$. Using the vanishing viscosity approximation, we obtain a sequence of functions, say $\left(u_{\varepsilon}\right)$, which converges toward an entropy admissible solution to (1.2), (1.3) (the initial data can be an arbitrary bounded function), but we cannot write down explicit formulas for $\left(u_{\varepsilon}\right)$. Using the weak asymptotic method, we have more freedom in choosing the regularization terms, and we are able to obtain explicit 
formulas for the appropriate sequence of solutions to the regularized problem, but, first, we cannot be sure that the sequence will converge toward an entropy admissible solution of (1.2), (1.3), and second, we need additional assumptions on the flux and initial data. In order to overcome the first obstacle, we start from Lipschitz continuous initial data and construct a solution along characteristics. In the case considered in this contribution, we have the possibility of choosing an appropriate regularization which enables us to modify characteristics so that they become nonintersecting but still to approximate the process described by the conservation law accurately enough. Thus, we will necessarily obtain an entropy admissible solution in the limit (see Theorem 3.3).

The following theorem defines so called "switch" functions $B_{i}: \mathbf{R} \rightarrow(0,1), i=1,2$, which are very important objects in the method.

Theorem 2.3 (7, 3]). Suppose that the functions $\omega_{i} \in C^{\infty}(\mathbf{R}), i=1,2$, satisfy $\lim _{z \rightarrow+\infty} \omega_{i}(z)=1, \lim _{z \rightarrow-\infty} \omega_{i}(z)=0$ and $\frac{d \omega_{i}(z)}{d z} \in \mathcal{S}(\mathbf{R})$, where $\mathcal{S}(\mathbf{R})$ is the Schwartz space of rapidly decreasing functions.

The functions $B_{i} \in C^{\infty}(\mathbf{R}), i=1,2$, defined for any $\rho \in \mathbf{R}$ by

$$
B_{1}(\rho)=\int \dot{\omega}_{1}(z) \omega_{2}(z+\rho) d z \text { and } B_{2}(\rho)=\int \dot{\omega}_{2}(z) \omega_{1}(z-\rho) d z
$$

satisfy

$$
\begin{gathered}
B_{1}(\rho)+B_{2}(\rho)=1, \\
\lim _{\tau \rightarrow-\infty} \tau^{N} B_{1}(\tau)=0, \quad \lim _{\tau \rightarrow \infty} \tau^{N} B_{2}(\tau)=0 \Rightarrow \lim _{\tau \rightarrow \pm \infty} \tau^{N} B_{1}(\tau) B_{2}(\tau)=0 .
\end{gathered}
$$

The functions $\omega_{i}, i=1,2$, can be chosen so that

$$
B_{2}(z)=1-B_{1}(z) \equiv 0, \quad z<0 .
$$

In the sequel, by $t^{*}(s), s \in \mathbf{R}^{d-1}$, we denote the moment of intersection of characteristics (1.4) emanating from the fibre connecting $x=X(0, s)$ and $x=X\left(\hat{\tau}_{0}(s), s\right)$, $s \in \mathbf{R}^{d-1}$, respectively, where $X$ is given by (1.9). From (1.5) and (1.6), it follows that

$$
t^{*}(s)=\frac{1}{K(s)} \text {. }
$$

We shall also write

$$
\tau=\frac{t-t^{*}(s)}{\varepsilon}, \quad B_{i}=B_{i}\left(\frac{t-t^{*}(s)}{\varepsilon}\right), \quad \dot{B}_{i}=\left(B_{i}(\tau)\right)_{\tau}^{\prime}, \quad i=1,2 .
$$

Now, we can motivate the approximate problem that we shall solve. First, recall that our aim is to find a globally defined smooth approximate solution to (1.2), (1.3). To do this we have to avoid the intersection of characteristics. A natural idea is to smear the discontinuity line corresponding to every fibre, i.e. to take an $\varepsilon$-neighborhood of the discontinuity line and to dispose of characteristics in that neighborhood in a way that they do not intersect, but, as $\varepsilon \rightarrow 0$, all of them lump together into the discontinuity line. Along such lines, the approximate solution to our problem will remain constant. Such lines we call the "new characteristics" (see Figure 6).

In order to accomplish this idea, we shall use the switch functions $B_{i}, i=1,2$, from Theorem 2.3. Namely, notice that $B_{1}\left(\frac{t-t^{*}(s)}{\varepsilon}\right)=1-B_{2}\left(\frac{t-t^{*}(s)}{\varepsilon}\right)$ is close to zero for 


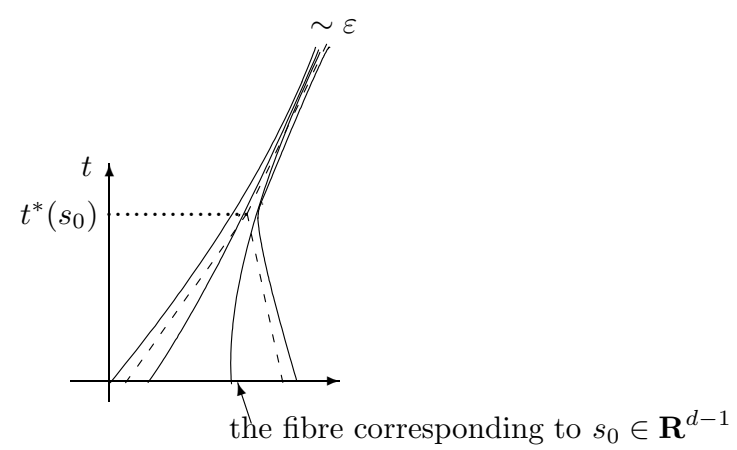

FIG. 6. The new characteristics issuing from the fibre corresponding to $s_{0} \in \mathbf{R}^{d-1}$. The distance between the rear most new characteristics is of order $\varepsilon$ for $t>t^{*}(s)$. The new characteristics are nonintersecting.

$t<t^{*}(s)$, and close to one for $t>t^{*}(s)$. Next, notice that for $t<t^{*}(s)$ we will have the classical solution to (1.2), (1.3), while for $t>t^{*}(s)$, a shock wave is formed. Therefore, it is natural to consider the following family of problems (compare with [8, Theorem 10]):

$$
\begin{aligned}
\partial_{t} u_{\varepsilon}+B_{2}\left(\frac{t-t^{*}(s)}{\varepsilon}\right)\left\langle\nabla, f\left(u_{\varepsilon}\right)\right\rangle+B_{1}\left(\frac{t-t^{*}(s)}{\varepsilon}\right) \sum_{i=1}^{d} c_{i} \frac{\partial u_{\varepsilon}}{\partial x_{i}} & =0, \\
\left.u_{\varepsilon}\right|_{t=0} & =u_{0}(x),
\end{aligned}
$$

where $s=s(x) \in R^{d-1}, x \in \mathbf{R}^{d}$, are the last $d-1$ variables of the inverse of the function $(\hat{\tau}, s) \mapsto X(\hat{\tau}, s)$ given by (1.9), and $c=\left(c_{1}, \ldots, c_{d}\right) \in \mathbf{R}^{d}$ is a constant to be determined in the next section. For simplicity, we shall also assume that $B_{i}, i=1,2$, satisfy (2.2).

Notice that for $t<t^{*}(s)$, we have $B_{2} \sim 1$ and $B_{1} \sim 0$, which means that (1.2) and (2.4) coincide in the direction of an appropriate fibre when $t<t^{*}(s)$. On the other hand, for $t>t^{*}(s)$, we have $B_{2} \sim 0$ and $B_{1} \sim 1$, which means that the nonlinear part of (2.4) disappears and the equation is governed by the linear part $\sum_{i=1}^{d} c_{i} \frac{\partial u_{\varepsilon}}{\partial x_{i}}$ in the direction of an appropriate fibre. It is well known that the linear part will not affect the profile of the corresponding initial data. In this case, the profile is a shock wave with the states $U_{L}$ and $U_{R}$, i.e. the function $u$ at the point of gradient catastrophe $\left(t^{*}(s), f^{\prime}\left(U_{L}\right) t^{*}(s)+\chi_{L}(s)\right) \in \mathbf{R}^{+} \times \mathbf{R}^{d}$. Since the variation $\left|U_{L}-U_{R}\right|$ is small, the form of a solution $u_{\varepsilon}$ to (2.4), (2.5) will also not have an influence on the formed shock wave.

We will find the weak asymptotic solution to problem (2.4), (2.5) by using the usual method of characteristics. Since the characteristics issuing from $\Omega_{L}$ and $\Omega_{R}$ bear the same information, we can allow their intersection, Thus, we are interested only in the characteristics issuing from $\Omega_{0}$.

The system of characteristics corresponding to (2.4), (2.5) has the following form:

$$
\begin{aligned}
\frac{d X_{i}}{d t} & =B_{2} f_{i}^{\prime}\left(u_{\varepsilon}\right)+B_{1} c_{i}, & X_{i}(0) & =x_{i 0}, \\
\frac{d u_{\varepsilon}}{d t} & =0, & u_{\varepsilon}(0) & =u_{1}\left(x_{0}\right), \quad x_{0} \in \Omega_{0},
\end{aligned}
$$


where $B_{i}=B_{i}\left(\frac{t-t^{*}(s)}{\varepsilon}\right), i=1,2$. It is easy to find the solution to the latter system:

$$
X\left(t, x_{0}\right)=x_{0}+f^{\prime}\left(u_{1}\right) \int_{0}^{t} B_{2} d t^{\prime}+c \int_{0}^{t} B_{1} d t^{\prime},
$$

where $B_{i}=B_{i}\left(\frac{t^{\prime}-t^{*}(s)}{\varepsilon}\right), i=1,2$. In order to show that the characteristics from (2.7) determine the solution to (2.4), (2.5) we need to prove that the Jacobian $J=\operatorname{det} \frac{\partial X}{\partial x_{0}}>0$ along the entire temporal axis. Indeed, if this is the case, then there exists the inverse function $x_{0}=x_{0}(X, t, \varepsilon)$ of the function $X$. Then, the classical solution to (2.4), (2.5) is given by $u_{1}\left(x_{0}(x, t, \varepsilon)\right)$ (again, we do not consider domains where the solution is constant).

According to assumptions (1.12), the transformation $x_{0}=x_{0}(\hat{\tau}, s)$ is regular. Therefore, in order to prove global solvability of (2.4), (2.5), it is enough to prove that $\operatorname{det}\left(\frac{\partial X}{\partial \tilde{\tau}}, \frac{\partial X}{\partial s}\right)>0$ along the entire temporal axis. We shall prove the latter fact under conditions which are necessarily fulfilled if $\left|U_{L}-U_{R}\right|$ is small enough (see Remark 2.5).

Lemma 2.4. Assume that $U_{L}$ and $U_{R}$ are such that for any $(\hat{\tau}, s) \in \dot{\bigcup}_{s \in \mathbf{R}^{d-1}}\left[0, \hat{\tau}_{0}(s)\right] \times\{s\}$ and any $t \in \mathbf{R}^{+}$,

$$
\operatorname{det}\left(\frac{\partial x_{0}}{\partial \hat{\tau}}, \frac{\partial x_{0}}{\partial s}+\left(f^{\prime}\left(u_{1}\right)-c\right) \nabla_{s} t^{*}(s) B_{1}\left(\frac{t-t^{*}(s)}{\varepsilon}\right)\right) \geq \alpha \operatorname{det}\left(\frac{\partial x_{0}}{\partial \hat{\tau}}, \frac{\partial x_{0}}{\partial s}\right),
$$

for an $\alpha>0$.

The Jacobian $\operatorname{det}\left(\frac{\partial X}{\partial \hat{\tau}}, \frac{\partial X}{\partial s}\right)$ of the characteristics (2.7) computed with respect to the variables $(\hat{\tau}, s)$ satisfies:

$$
\operatorname{det}\left(\frac{\partial X}{\partial \hat{\tau}}, \frac{\partial X}{\partial s}\right)>0, \quad(\hat{\tau}, s) \in \bigcup_{s \in \mathbf{R}^{d-1}}\left[0, \hat{\tau}_{0}(s)\right] \times\{s\} .
$$

Proof. Consider the first column of the matrix $\left(\frac{\partial X}{\partial \hat{\tau}}, \frac{\partial X}{\partial s}\right)$, where $X$ is given by (2.7),

$$
\frac{\partial X}{\partial \hat{\tau}}=\frac{\partial x_{0}}{\partial \hat{\tau}}+f^{\prime \prime}\left(u_{1}\right) \frac{\partial u_{1}}{\partial \hat{\tau}} \int_{0}^{t} B_{2} d t^{\prime}
$$

where we abbreviate $B_{2}=B_{2}\left(\frac{t^{\prime}-t^{*}(s)}{\varepsilon}\right)$. According to (1.8), we have for $x_{0} \in \Omega_{0}$ :

$$
\frac{\partial u_{1}\left(x_{0}\right)}{\partial \hat{\tau}}=-K(s), \quad \frac{\partial x_{0}}{\partial \hat{\tau}}=f^{\prime \prime}\left(u_{1}\right),
$$

and thus, from (2.10):

$$
\frac{\partial X}{\partial \hat{\tau}}=\left(1-K(s) \int_{0}^{t} B_{2} d t^{\prime}\right) \frac{\partial x_{0}}{\partial \hat{\tau}} .
$$

Notice that since we assumed $B_{2}(z)=0, z \leq 0$ (see (2.2)), it follows that

$$
1-K(s) \int_{0}^{t} B_{2} d t^{\prime} \geq 1-K(s) \int_{0}^{t *} B_{2} d t^{\prime}>0
$$

since $t^{*}(s)=1 / K(s)$ and $B_{2}(z)<1$ on a nonzero subset of $(-\infty, 0)$. 
Now, we pass to the columns $\frac{\partial X}{\partial s}$. From (2.7), we have

$$
\begin{aligned}
\frac{\partial X}{\partial s}=\frac{\partial x_{0}}{\partial s} & +f^{\prime \prime}\left(u_{1}\right) \frac{\partial u_{1}}{\partial s} \int_{0}^{t} B_{2} d t^{\prime} \\
& +f^{\prime}\left(u_{1}\right) \nabla_{s} t^{*}(s) \int_{0}^{t} \frac{1}{\varepsilon} \dot{B}_{2} d t^{\prime}+c \nabla_{s} t^{*}(s) \int_{0}^{t} \frac{1}{\varepsilon} \dot{B}_{1} d t^{\prime} .
\end{aligned}
$$

From here, having in mind the change of variables $\frac{t^{\prime}-t^{*}(s)}{\varepsilon}=z \Longrightarrow d t^{\prime}=\varepsilon d z$ and using $\dot{B}_{2}=-\dot{B}_{1}$, we obtain

$$
\frac{\partial X}{\partial s}=\frac{\partial x_{0}}{\partial s}+f^{\prime \prime}\left(u_{1}\right) \frac{\partial u_{1}}{\partial s} \int_{0}^{t} B_{2} d t-f^{\prime}\left(u_{1}\right) \nabla_{s} t^{*}(s) B_{1}+c \nabla_{s} t^{*}(s) B_{1} .
$$

Now, we are able to estimate the determinant $J=\operatorname{det}\left(\frac{\partial x}{\partial \hat{\tau}}, \frac{\partial x}{\partial s}\right)$. We have from (2.11), (2.12) and (2.13):

$$
\begin{aligned}
J & =\operatorname{det}\left(\frac{\partial X}{\partial \hat{\tau}}, \frac{\partial X}{\partial s}\right) \\
& =\left(1-\int_{0}^{t} B_{2} d t^{\prime}\right) \operatorname{det}\left(f^{\prime \prime}\left(u_{1}\right), \frac{\partial x_{0}}{\partial s}+f^{\prime \prime}\left(u_{1}\right) \frac{\partial u_{1}}{\partial s} \int_{0}^{t} B_{2} d t^{\prime}-\left(f^{\prime}\left(u_{1}\right)-c\right) \nabla_{s} t^{*}(s) B_{1}\right) \\
& =\left(1-\int_{0}^{t} B_{2} d t^{\prime}\right) \operatorname{det}\left(f^{\prime \prime}\left(u_{1}\right), \frac{\partial x_{0}}{\partial s}-\left(f^{\prime}\left(u_{1}\right)-c\right) \nabla_{s} t^{*}(s) B_{1}\right) \\
& \stackrel{(2.8)}{\geq} \alpha\left(1-\int_{0}^{t} B_{2} d t^{\prime}\right) \operatorname{det}\left(\frac{\partial x_{0}}{\partial \hat{\tau}}, \frac{\partial x_{0}}{\partial s}\right)>0
\end{aligned}
$$

according to (1.12) and (2.12).

This concludes the proof.

REMARK 2.5. Notice that, by relying on the continuity of the function det, the boundedness of the functions $\nabla_{s} t^{*}$ and $B_{1}$, the fact that $U_{R}<u_{1}<U_{L}$, and since we assumed $\operatorname{det}\left(\frac{\partial x_{0}}{\partial \tilde{\tau}}, \frac{\partial x_{0}}{\partial s}\right) \geq c>0$, if $\left|U_{L}-U_{R}\right|$ are sufficiently small, then (2.8) is satisfied. Indeed, notice that

$$
\begin{aligned}
f^{\prime}\left(u_{1}\right)-c & =f^{\prime}\left(u_{1}\right)-\frac{f\left(U_{L}\right)-f\left(U_{R}\right)}{U_{L}-U_{R}}=f^{\prime}\left(u_{1}\right)-\left(f_{1}^{\prime}\left(\tilde{u}_{1}\right), f_{2}^{\prime}\left(\tilde{u}_{2}\right), \ldots, f_{d}^{\prime}\left(\tilde{u}_{d}\right)\right) \\
& =\left(\left(u_{1}-\tilde{u}_{1}\right) f_{1}^{\prime \prime}\left(\hat{u}_{1}\right),\left(u_{1}-\tilde{u}_{2}\right) f_{2}^{\prime \prime}\left(\hat{u}_{2}, \ldots,\left(u_{1}-\tilde{u}_{d}\right) f_{d}^{\prime \prime}\left(\hat{u}_{d}\right)\right)\right.
\end{aligned}
$$

for values $\tilde{u}_{i} \in\left[U_{R}, U_{L}\right]$ and $\hat{u}_{i} \in\left[\min \left\{u_{i}, \tilde{u}_{i}\right\}, \max \left\{u_{i}, \tilde{u}_{i}\right\}\right] \subset\left[U_{R}, U_{L}\right], i=1, \ldots, d$, according to the Lagrange mean value theorem. From here, it further follows that

$$
\left\|f^{\prime}\left(u_{1}\right)-c\right\| \leq C\left|U_{L}-U_{R}\right|,
$$

where $\|\cdot\|$ denotes the Euclidean norm and $C$ a constant such that $C \geq \max _{U_{R} \leq u \leq U_{R}}\left|f^{\prime \prime}(u)\right|$. Now, using continuity of the function det, we conclude that

$$
\begin{aligned}
& \operatorname{det}\left(\frac{\partial x_{0}}{\partial \hat{\tau}}, \frac{\partial x_{0}}{\partial s}+\left(f^{\prime}\left(u_{1}\right)-c\right) \nabla_{s} t^{*}(s) B_{1}\left(\frac{t-t^{*}(s)}{\varepsilon}\right)\right) \\
& =\operatorname{det}\left(\frac{\partial x_{0}}{\partial \hat{\tau}}, \frac{\partial x_{0}}{\partial s}\right)+\mathcal{O}\left(\left\|f^{\prime}\left(u_{1}\right)-c\right\|\right),
\end{aligned}
$$


since $B_{1}$ and $\nabla_{s} t^{*}$ are bounded functions. From here and (2.15), we conclude that

$$
\begin{aligned}
& \operatorname{det}\left(\frac{\partial x_{0}}{\partial \hat{\tau}}, \frac{\partial x_{0}}{\partial s}+\left(f^{\prime}\left(u_{1}\right)-c\right) \nabla_{s} t^{*}(s) B_{1}\left(\frac{t-t^{*}(s)}{\varepsilon}\right)\right) \\
& =\operatorname{det}\left(\frac{\partial x_{0}}{\partial \hat{\tau}}, \frac{\partial x_{0}}{\partial s}\right)+\left|U_{L}-U_{R}\right| \mathcal{O}(1) .
\end{aligned}
$$

Since, according to (1.12), $\operatorname{det}\left(\frac{\partial x_{0}}{\partial \hat{\tau}}, \frac{\partial x_{0}}{\partial s}\right) \geq c>0$, it follows that for any $0<\alpha<1$ we can choose $\left|U_{L}-U_{R}\right|$ small enough so that $\left|U_{L}-U_{R}\right| \mathcal{O}(1)<\alpha \operatorname{det}\left(\frac{\partial x_{0}}{\partial \hat{\tau}}, \frac{\partial x_{0}}{\partial s}\right)$. For such chosen $U_{l}$ and $U_{R}$, we have from (2.16):

$$
\operatorname{det}\left(\frac{\partial x_{0}}{\partial \hat{\tau}}, \frac{\partial x_{0}}{\partial s}+\left(f^{\prime}\left(u_{1}\right)-c\right) \nabla_{s} t^{*}(s) B_{1}\left(\frac{t-t^{*}(s)}{\varepsilon}\right)\right)>(1-\alpha) \operatorname{det}\left(\frac{\partial x_{0}}{\partial \hat{\tau}}, \frac{\partial x_{0}}{\partial s}\right),
$$

implying that (2.8) is satisfied.

In the sequel, we denote by $\Omega_{0}^{t}$ the set $\Omega_{0}$ shifted along the new characteristics $X$ from (2.7) for the time $t$ (at the level $t$ ), i.e.

$$
\Omega_{0}^{t}=\left\{y \in \mathbf{R}^{d}: y=X\left(x_{0}, t, \varepsilon\right), \quad x_{0} \in \Omega_{0}\right\} .
$$

Furthermore, by $\Omega_{L}^{t}$ we denote the set $\Omega_{L}$ shifted along the standard characteristics $x=f^{\prime}\left(U_{L}\right) t+x_{0}, x_{0} \in \Omega_{L}$, until the intersection with $\Omega_{0}^{t}$. More precisely,

$$
\Omega_{L}^{t}=\left\{y \in \mathbf{R}^{d}: y=f^{\prime}\left(U_{L}\right) t+x_{0}, x_{0} \in \Omega_{L}, \text { and } \sharp t^{\prime} \in[0, t], f^{\prime}\left(U_{L}\right) t^{\prime}+x_{0} \in \Omega_{0}^{t^{\prime}}\right\} .
$$

Similarly, we let:

$$
\Omega_{R}^{t}=\left\{y \in \mathbf{R}^{d}: y=f^{\prime}\left(U_{R}\right) t+x_{0}, x_{0} \in \Omega_{R}, \text { and } ¥ t^{\prime} \in[0, t], f^{\prime}\left(U_{R}\right) t^{\prime}+x_{0} \in \Omega_{0}^{t^{\prime}}\right\} .
$$

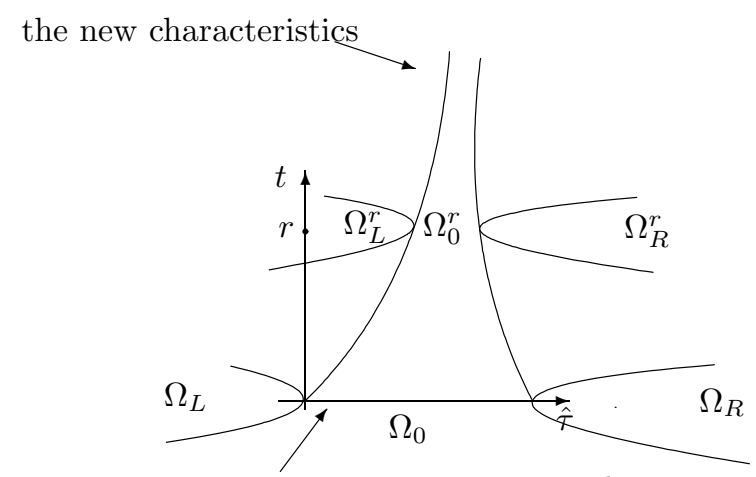

the fibre corresponding to $s_{0} \in \mathbf{R}^{d-1}$

FIG. 7. $\Omega_{0}^{r}, \Omega_{L}^{r}$ and $\Omega_{R}^{r}$ are the sets $\Omega_{0}, \Omega_{L}$ and $\Omega_{R}$ moved along the new characteristics at the level $t=r$.

Notice that, since the characteristics are nonintersecting, we have

$$
\mathbf{R}^{d}=\overline{\Omega_{0}^{t}} \dot{\cup} \Omega_{L}^{t} \dot{\cup} \Omega_{R}^{t}
$$

A simple corollary of Lemma 2.4 is the existence of the weak asymptotic solution to the Cauchy problem (2.4), (2.5): 
Corollary 2.6. There exists a weak asymptotic solution to (2.4), (2.5).

Proof. It follows from Lemma 2.4 that there exists an inverse function $x_{0}(t, x, \varepsilon)$, $x \in \mathbf{R}^{d}$, of the function $X\left(x_{0}, t, \varepsilon\right), x_{0} \in \Omega_{0}$, defined by (2.7).

Thus, the weak asymptotic solution to (2.4), (2.5) is given by:

$$
u_{\varepsilon}(x, t)= \begin{cases}U_{L}, & x \in \Omega_{L}^{t}, \\ u_{1}\left(x_{0}(t, x, \varepsilon)\right), & x \in \Omega_{t}^{0}, \\ U_{R}, & x \in \Omega_{R}^{t} .\end{cases}
$$

3. The weak asymptotic equivalence. We will show that the weak asymptotic solution to (2.4), (2.5) is, at the same time, the weak asymptotic solution to (1.2), (1.3). More precisely, the following theorem holds.

Theorem 3.1. Let $\left(u_{\varepsilon}\right)$ be the weak asymptotic solution to (2.4), (2.5). Then:

$$
\partial_{t} u_{\varepsilon}+B_{2}\left\langle\nabla, f\left(u_{\varepsilon}\right)\right\rangle+B_{1} \sum_{i=1}^{d} c_{i} \frac{\partial u_{\varepsilon}}{\partial x_{i}}=\partial_{t} u_{\varepsilon}+\left\langle\nabla, f\left(u_{\varepsilon}\right)\right\rangle+o_{\mathcal{D}^{\prime}}(1), \quad \varepsilon \rightarrow 0 .
$$

Proof. Since $\left\langle\nabla, f\left(u_{\varepsilon}\right)\right\rangle=\left(B_{1}+B_{2}\right)\left\langle\nabla, f\left(u_{\varepsilon}\right)\right\rangle$, (3.1) is equivalent to

$$
B_{1}\left\langle\nabla, f\left(u_{\varepsilon}(t, \cdot)\right)-c u_{\varepsilon}(t, \cdot)\right\rangle=o_{\mathcal{D}^{\prime}}(1) .
$$

We multiply this by $\varphi \in C_{0}^{1}\left(\mathbf{R}^{d}\right)$ and integrate over $\mathbf{R}^{d}$. We get after standard integration by parts (the Gauss-Ostrogradskii formula):

$$
\begin{aligned}
& \int_{\mathbf{R}^{d}} B_{1}\left\langle\nabla, f\left(u_{\varepsilon}(t, x)\right)-c u_{\varepsilon}(t, x)\right\rangle \varphi d x \\
& =-\int_{\mathbf{R}^{d}}\left\langle\nabla\left(B_{1} \varphi\right), f\left(u_{\varepsilon}(t, x)\right)-c u_{\varepsilon}(t, x)\right\rangle d x \\
& =-\int_{\Omega_{L}^{t}}\left\langle\nabla\left(B_{1} \varphi\right), f\left(U_{L}\right)-c U_{L}\right\rangle d x-\int_{\Omega_{R}^{t}}\left\langle\nabla\left(B_{1} \varphi\right), f\left(U_{R}\right)-c U_{R}\right\rangle d x \\
& -\int_{\Omega_{0}^{t}}\left\langle\nabla\left(B_{1} \varphi\right), f\left(u_{\varepsilon}(t, x)\right)-c u_{\varepsilon}(t, x)\right\rangle d x=o(1) .
\end{aligned}
$$

Consider the integral in the latter formula corresponding to the domain $\Omega_{R}^{t}$. Denote by $\left(\Omega_{R}^{t}\right)^{C}$ the complement of the set $\Omega_{R}^{t}$. Notice that $\left(\Omega_{R}^{t}\right)^{C}=\Omega_{L}^{t} \cup \Omega_{0}^{t}$ and, using integration by parts again,

$$
\begin{aligned}
& \int_{\Omega_{R}^{t}}\left\langle\nabla\left(B_{1} \varphi\right), f\left(U_{R}\right)-c U_{R}\right\rangle d x=\int_{\partial \Omega_{R}^{t}}\left\langle B_{1} \varphi\left(f\left(U_{R}\right)-c U_{R}\right), \vec{n}\right\rangle d s \\
& =-\int_{\left(\Omega_{R}^{t}\right)^{C}}\left\langle\nabla\left(B_{1} \varphi\right), f\left(U_{R}\right)-c U_{R}\right\rangle d x \\
& =-\int_{\Omega_{L}^{t}}\left\langle\nabla\left(B_{1} \varphi\right), f\left(U_{R}\right)-c U_{R}\right\rangle d x-\int_{\Omega_{0}^{t}}\left\langle\nabla\left(B_{1} \varphi\right), f\left(U_{R}\right)-c U_{R}\right\rangle d x .
\end{aligned}
$$


We conclude from (3.2) and (3.3):

$$
\begin{aligned}
& \int_{\mathbf{R}^{d}} B_{1}\left\langle\nabla, f\left(u_{\varepsilon}(t, x)\right)-c u_{\varepsilon}(t, x)\right\rangle \varphi d x \\
& =-\int_{\Omega_{L}^{t}}\left\langle\nabla\left(B_{1} \varphi\right), f\left(U_{L}\right)-f\left(U_{R}\right)-c\left(U_{L}-U_{R}\right)\right\rangle d x \\
& \quad+\int_{\Omega_{0}^{t}}\left\langle\nabla\left(B_{1} \varphi\right), f\left(u_{\varepsilon}(t, x)\right)-f\left(U_{R}\right)-c\left(u_{\varepsilon}(t, x)-U_{R}\right)\right\rangle d x=o(1) .
\end{aligned}
$$

Choosing here

$$
c=\left(\frac{f_{1}\left(U_{R}\right)-f_{1}\left(U_{L}\right)}{U_{R}-U_{L}}, \ldots, \frac{f_{d}\left(U_{R}\right)-f_{d}\left(U_{L}\right)}{U_{R}-U_{L}}\right),
$$

we conclude that (3.2) is equivalent to

$$
\begin{aligned}
& \int_{\mathbf{R}^{d}} B_{1}\left\langle\nabla, f\left(u_{\varepsilon}(t, x)\right)-c u_{\varepsilon}(t, x)\right\rangle \varphi d x \\
& =\int_{\Omega_{0}^{t}}\left\langle\nabla\left(B_{1} \varphi\right), f\left(u_{\varepsilon}(t, x)\right)-f\left(U_{R}\right)-c\left(u_{\varepsilon}(t, x)-U_{R}\right)\right\rangle d x=o(1) .
\end{aligned}
$$

Let us prove the second equality in (3.5). Denote for simplicity

$$
G\left(u_{\varepsilon}(t, x)\right)=f\left(u_{\varepsilon}(t, x)\right)-f\left(U_{R}\right)-c\left(u_{\varepsilon}(t, x)-U_{R}\right) .
$$

Then

$$
\begin{aligned}
& \int_{\Omega_{0}^{t}}\left\langle\nabla\left(B_{1} \varphi\right), G\left(u_{\varepsilon}(t, x)\right)\right\rangle d x \\
& =\int_{\Omega_{0}^{t}}\left\langle B_{1} \nabla \varphi, G\left(u_{\varepsilon}(t, x)\right)\right\rangle d x-\int_{\Omega_{0}^{t}} \varphi \frac{\dot{B}_{1}}{\varepsilon}\left\langle\nabla t^{*}(s), G\left(u_{\varepsilon}(t, x)\right)\right\rangle d x \\
& =\int_{\Omega_{0}^{t}}\left\langle B_{1} \nabla \varphi, G\left(u_{\varepsilon}(t, x)\right)\right\rangle d x-\int_{\Omega_{0}^{t}} \varphi \frac{\dot{B}_{1}}{\varepsilon}\left\langle\nabla_{s} t^{*}(s) \frac{\partial s}{\partial x}, G\left(u_{\varepsilon}(t, x)\right)\right\rangle d x .
\end{aligned}
$$

For the function $X\left(t, x_{0}(\hat{\tau}, s), \varepsilon\right)$ from (2.7), introduce the following change of variables in the latter integrals:

$$
X=X\left(t, x_{0}(\hat{\tau}, s), \varepsilon\right) \Longrightarrow \operatorname{det}\left(\frac{\partial X}{\partial \hat{\tau}}, \frac{\partial X}{\partial s}\right) \stackrel{[2.14]}{=}\left(1-K(s) \int_{0}^{t} B_{2} d t^{\prime}\right) \operatorname{det}\left(\frac{\partial x_{0}}{\partial \hat{\tau}}, \frac{\partial x_{0}}{\partial s}\right),
$$

where $B_{2}=B_{2}\left(\frac{t^{\prime}-t^{*}(s)}{\varepsilon}\right)$. To proceed, it is convenient to notice the following:

$$
\begin{aligned}
1-K(s) \int_{0}^{t} B_{2} d t^{\prime} & =1-K(s)\left(\int_{0}^{t^{*}(s)} B_{2} d t^{\prime}+\int_{t^{*}(s)}^{t} B_{2} d t^{\prime}\right) \\
& =1-K(s) \int_{0}^{t^{*}(s)}\left(1-B_{1}\right) d t^{\prime}-K(s) \int_{t^{*}(s)}^{t} B_{2} d t^{\prime} \\
& =1-K(s) t^{*}(s)+K(s)\left(\int_{0}^{t *(s)} B_{1} d t^{\prime}+\int_{t^{*}(s)}^{t} B_{2} d t^{\prime}\right) \\
& =K(s)\left(\varepsilon \int_{-t^{*}(s) / \varepsilon}^{0} B_{1}(z) d z+\varepsilon \frac{\tau}{t-t^{*}(s)} \int_{t^{*}(s)}^{t} B_{2} d t^{\prime}\right),
\end{aligned}
$$


where in the last step we introduced the change of variables $\frac{t^{\prime}-t^{*}(s)}{\varepsilon}=z$, and, as usual, denoted $\tau=\frac{t-t^{*}(s)}{\varepsilon}$. Thus, from here and (3.7), we conclude that

$$
\operatorname{det}\left(\frac{\partial X}{\partial \hat{\tau}}, \frac{\partial X}{\partial s}\right)=\varepsilon \tau \mathcal{O}(1) \operatorname{det}\left(\frac{\partial x_{0}}{\partial \hat{\tau}}, \frac{\partial x_{0}}{\partial s}\right), \quad \tau \rightarrow \infty
$$

We get from here and (3.6) (the $\cdot$ in the formula below stands for $x_{0}(\hat{\tau}, s)$ for $x_{0}$ from $(1.12)$ :

$$
\begin{aligned}
& \int_{\Omega_{0}^{t}}\left\langle\nabla\left(B_{1} \varphi\right), G\left(u_{\varepsilon}(t, x)\right)\right\rangle d x \\
& \stackrel{\text { 3.8) }}{=} \int_{\Gamma_{L}} \int_{0}^{\hat{\tau}_{0}(s)} \varepsilon \tau \mathcal{O}(1) B_{1}\left\langle\nabla \varphi, G\left(u_{1}\left(x_{0}(t, \cdot, \varepsilon)\right)\right)\right\rangle \operatorname{det}\left(\frac{\partial x_{0}}{\partial \hat{\tau}}, \frac{\partial x_{0}}{\partial s}\right) d \hat{\tau} d s \\
& \quad+\int_{\Gamma_{L}} \int_{0}^{\hat{\tau}_{0}(s)} \tau \mathcal{O}(1) \dot{B}_{1} \varphi\left\langle\nabla_{s} t^{*}(s) \frac{\partial s}{\partial x}, G\left(u_{1}\left(x_{0}(t, \cdot, \varepsilon)\right)\right)\right\rangle \operatorname{det}\left(\frac{\partial x_{0}}{\partial \hat{\tau}}, \frac{\partial x_{0}}{\partial s}\right) d \hat{\tau} d s \\
& =\mathcal{O}(\varepsilon)+\int_{\Gamma_{L}} \int_{0}^{\hat{\tau}_{0}(s)} \tau \mathcal{O}(1) \dot{B}_{1} \varphi\left\langle\nabla_{s} t^{*}(s) \frac{\partial s}{\partial x}, G\left(u_{1}\left(x_{0}(t, \cdot, \varepsilon)\right)\right)\right\rangle \times \operatorname{det}\left(\frac{\partial x_{0}}{\partial \hat{\tau}}, \frac{\partial x_{0}}{\partial s}\right) d \hat{\tau} d s
\end{aligned}
$$

where, as usual, $B_{i}=B_{i}\left(\frac{t-t^{*}(s)}{\varepsilon}\right)=B_{i}(\tau), i=1,2$. So, we see that it remains to estimate

$$
\begin{aligned}
& \int_{\Gamma_{L}} \int_{0}^{\hat{\tau}_{0}(s)} \tau \mathcal{O}(1) \dot{B}_{1} \varphi\left\langle\nabla_{s} t^{*}(s) \frac{\partial s}{\partial x}, G\left(u_{1}\left(x_{0}(t, \cdot, \varepsilon)\right)\right)\right\rangle \operatorname{det}\left(\frac{\partial x_{0}}{\partial \hat{\tau}}, \frac{\partial x_{0}}{\partial s}\right) d \hat{\tau} d s \\
& =\int_{\left|\nabla_{s} t^{*}\right| \leq \varepsilon} \int_{0}^{\hat{\tau}_{0}(s)} \tau \mathcal{O}(1) \dot{B}_{1} \varphi\left\langle\nabla_{s} t^{*}(s) \frac{\partial s}{\partial x}, G\left(u_{1}\left(x_{0}(t, \cdot, \varepsilon)\right)\right)\right\rangle \operatorname{det}\left(\frac{\partial x_{0}}{\partial \hat{\tau}}, \frac{\partial x_{0}}{\partial s}\right) d \hat{\tau} d s \\
& +\int_{\left|\nabla_{s} t^{*}\right| \geq \varepsilon} \tau \mathcal{O}(1) \dot{B}_{1} \varphi\left\langle\nabla_{s} t^{*}(s) \frac{\partial s}{\partial x}, G\left(u_{1}\left(x_{0}(t, \cdot, \varepsilon)\right)\right)\right\rangle \operatorname{det}\left(\frac{\partial x_{0}}{\partial \hat{\tau}}, \frac{\partial x_{0}}{\partial s}\right) d \hat{\tau} d s
\end{aligned}
$$

Since

$$
\int_{\left|\nabla_{s} t^{*}\right| \leq \varepsilon} \int_{0}^{\hat{\tau}_{0}(s)} \tau \mathcal{O}(1) \dot{B}_{1} \varphi\left\langle\nabla_{s} t^{*}(s) \frac{\partial s}{\partial x}, G\left(u_{1}\left(x_{0}(t, \cdot, \varepsilon)\right)\right)\right\rangle \operatorname{det}\left(\frac{\partial x_{0}}{\partial \hat{\tau}}, \frac{\partial x_{0}}{\partial s}\right) d \hat{\tau} d s=\mathcal{O}(\varepsilon)
$$

we only need to estimate the last term on the right-hand side of (3.10). First assume, without losing generality, that if $\left|\nabla_{s} t^{*}(s)\right| \geq \varepsilon$, then for a fixed $k \in\{1, \ldots, d-1\}$,

$$
\frac{\partial_{s_{i}} t^{*}(s)}{\partial_{s_{k}} t^{*}(s)} \leq C<\infty, \quad i=1, \ldots, d-1
$$

It is clear that the latter holds at least locally, which is enough for further consideration (we would involve the partition of unity argument if $k$ would be different for different neighborhoods).

Then, take the following change of variables:

$$
y_{k}=\frac{t-t^{*}(s)}{\varepsilon}=\tau, \quad y_{i}=s_{i}, i \neq k \Longrightarrow \operatorname{det}\left(\frac{\partial s}{\partial y}\right)=\frac{\varepsilon}{\partial_{s_{k}} t^{*}(s(y))},
$$


and denote $Y=y\left(\left\{s:\left|\nabla t^{*}(s)\right| \geq \varepsilon\right\}\right)$. Then

$$
\begin{aligned}
& \int_{\left|\nabla t^{*}\right| \geq \varepsilon} \int_{0}^{\hat{\tau}_{0}(s)} \tau \mathcal{O}(1) \dot{B}_{1} \varphi\left\langle\nabla_{s} t^{*}(s) \frac{\partial s}{\partial x}, G\left(u_{1}\left(x_{0}(t, \cdot, \varepsilon)\right)\right)\right\rangle \operatorname{det}\left(\frac{\partial x_{0}}{\partial \hat{\tau}}, \frac{\partial x_{0}}{\partial s}\right) d \hat{\tau} d s \\
& =\int_{Y} \int_{0}^{\hat{\tau}_{0}(s(y))} \varepsilon \tau \mathcal{O}(1) y_{k} \dot{B}_{1}\left(y_{k}\right) \varphi\left\langle\frac{\nabla t^{*}(s)}{\partial_{s_{k}} t^{*}(s)} \frac{\partial s}{\partial x}, G\left(u_{1}\left(x_{0}(t, \cdot, \varepsilon)\right)\right)\right\rangle \operatorname{det}\left(\frac{\partial x_{0}}{\partial \hat{\tau}}, \frac{\partial x_{0}}{\partial s}\right) d \hat{\tau} d y \\
& =\mathcal{O}(\varepsilon),
\end{aligned}
$$

according to (3.12).

Noticing that $(3.13),(3.11) \Longrightarrow(3.9) \Longrightarrow(3.5) \Longrightarrow(3.2)$, the proof of the theorem is completed.

The last issue that we need to deal with is the entropy admissibility of the weak solution to (1.2), (1.3), which is obtained as a limit of the weak asymptotic solution. As expected, for a solution constructed from regular initial data along the characteristics, the solution obtained as a limit of the weak asymptotic solution is admissible (since characteristics must run into the shock surface; see the Lax-Oleinik admissibility conditions [2]). First, let us recall the notion of the entropy admissible weak solution introduced by S. N. Kruzhkov in his famous paper [20].

Definition 3.2. We say that a weak solution to (1.2), (1.3) is entropy admissible if for every nonnegative $\varphi \in C_{0}^{\infty}\left(\mathbf{R}^{+} \times \mathbf{R}^{d}\right)$ and every $\lambda \in \mathbf{R}$, it follows that

$$
\left.\iint_{\mathbf{R}^{\times} \mathbf{R}^{d}} \operatorname{sgn}_{ \pm}(u-\lambda)\left((u-\lambda) \frac{\partial \varphi}{\partial t}+\sum_{i=1}^{d}\left(f_{i}(u)-f_{i}(\lambda)\right) \frac{\partial \varphi}{\partial x_{i}}\right)\right) d x d t \geq 0,
$$

where

$$
\operatorname{sgn}_{+}(u-\lambda)=\left(|u-\lambda|^{+}\right)_{u}^{\prime}= \begin{cases}1, & u>\lambda \\ 0, & u \leq \lambda\end{cases}
$$

and

$$
\operatorname{sgn}_{-}(u-\lambda)=\left(|u-\lambda|^{-}\right)_{u}^{\prime}= \begin{cases}0, & u>\lambda, \\ -1, & u \leq \lambda .\end{cases}
$$

The following theorem holds.

Theorem 3.3. Assume that $0<U_{L}-U_{R}<\delta$ for a small enough constant $\delta$. Then, the weak asymptotic solution $\left(u_{\varepsilon}\right)$ to (1.2), (1.3) is given by (2.17). For every $s \in \mathbf{R}^{d-1}$ and $t \geq t^{*}(s)$, the a.e. pointwise limit of the weak asymptotic solution $\left(u_{\varepsilon}\right)$ to problem (1.2), (1.3) contains an admissible shock wave of the strength $\left|U_{L}-U_{R}\right|$ which is formed at the point $X=f^{\prime}\left(U_{L}\right) t^{*}(s)+\chi_{L}(s)$ at the moment $t=t^{*}(s)$. The corresponding shock surface moves by the law

$$
x=f^{\prime}\left(U_{L}\right) t^{*}(s)+\chi_{L}(s)+\frac{f\left(U_{L}\right)-f\left(U_{R}\right)}{U_{L}-U_{R}}\left(t-t^{*}(s)\right) .
$$

Proof. From (1.6), we see that the initial data (1.3) corresponding to equation (1.2) will evolve into the shock wave at the moment $t^{*}(s)=\frac{1}{K(s)}($ see (1.5) and (1.6)). More precisely, any characteristic issuing from the point $x_{0}=x_{0}(\hat{\tau}, s) \in \Omega_{0}$, where the function 
$x_{0}$ is given by (1.11), will enter the shock surface at the moment $t^{*}(s)$. The shock surface then continues to move by the law

$$
x=f^{\prime}\left(U_{L}\right) t^{*}(s)+\chi_{L}(s)+\frac{f\left(U_{L}\right)-f\left(U_{R}\right)}{U_{L}-U_{R}}\left(t-t^{*}(s)\right)
$$

which is obtained by letting $\varepsilon \rightarrow 0$ in (2.7) for $t>t^{*}(s)$.

Next, notice that from the form of the weak asymptotic solution (2.17) and Theorem 3.1] it follows that it converges pointwisely a.e. toward a weak solution $u \in L^{\infty}\left(\mathbf{R}^{+} \times \mathbf{R}^{d}\right)$ to (1.2), (1.3). Actually, in a neighborhood of any $(t, x) \in \mathbf{R}^{+} \times \mathbf{R}^{d}$, the function $u$ will be either the shock wave with values $U_{L}$ and $U_{R}$ (if $x_{0}(t, x, \varepsilon)=x_{0}(\hat{\tau}, s)$ such that $t \geq t^{*}(s)$, where $x_{0}$ is given by (1.11) $)$, or it will be the classical solution to (1.2), (1.3) (if $x_{0}(t, x, \varepsilon)=x_{0}(\hat{\tau}, s)$ such that $t<t^{*}(s)$, where $x_{0}$ is given by (1.11)).

We shall prove that the weak solution $u$ is entropy admissible in the sense of Definition 3.2 for the entropies $|\cdot-\lambda|^{+}$(the situation with $|\cdot-\lambda|^{-}$is analogical). Since $u_{\varepsilon}$ are the Lipschitz continuous functions, from (2.4) we conclude that for an arbitrary $\varphi \in$ $C_{0}^{1}\left(\mathbf{R}^{+} \times \mathbf{R}^{d}\right)$, it follows that

$$
\begin{aligned}
& \iint_{\mathbf{R}^{+} \times \mathbf{R}^{d}} \operatorname{sgn}_{+}\left(u_{\varepsilon}-\lambda\right)\left(\left(u_{\varepsilon}-\lambda\right) \frac{\partial \varphi}{\partial t}+\sum_{i=1}^{d}\left(f_{i}\left(u_{\varepsilon}\right)-f_{i}(\lambda)\right) \frac{\partial \varphi}{\partial x_{i}}\right) d x d t \\
& =\iint_{\mathbf{R}^{+} \times \mathbf{R}^{d}} \operatorname{sgn}_{+}\left(u_{\varepsilon}-\lambda\right)\left(\left(u_{\varepsilon}-\lambda\right) \frac{\partial \varphi}{\partial t}\right. \\
& \left.+\sum_{i=1}^{d}\left(\left(f_{i}\left(u_{\varepsilon}\right)-f_{i}(\lambda)\right) \frac{\partial\left(B_{2} \varphi\right)}{\partial x_{i}}+c_{i}\left(u_{\varepsilon}-\lambda\right) \frac{\partial\left(B_{1} \varphi\right)}{\partial x_{i}}\right)\right) d x d t \\
& +\iint_{\mathbf{R}^{+} \times \mathbf{R}^{d}} \operatorname{sgn}_{+}\left(u_{\varepsilon}-\lambda\right) \sum_{i=1}^{d}\left(\left(f_{i}\left(u_{\varepsilon}\right)-f_{i}(\lambda)\right)-c_{i}\left(u_{\varepsilon}-\lambda\right)\right) \frac{\partial\left(B_{1} \varphi\right)}{\partial x_{i}} d x d t \\
& =\iint_{\mathbf{R}^{+} \times \mathbf{R}^{d}} \operatorname{sgn}_{+}(u-\lambda) \sum_{i=1}^{d}\left(\left(f_{i}\left(u_{\varepsilon}\right)-f_{i}(\lambda)\right)-c_{i}\left(u_{\varepsilon}-\lambda\right)\right) \frac{\partial\left(B_{1} \varphi\right)}{\partial x_{i}} d x d t
\end{aligned}
$$

where $c_{i}=\frac{\left[f_{i}\right]}{[u]}=\frac{f_{i}\left(U_{L}\right)-f_{i}\left(U_{R}\right)}{U_{L}-U_{R}}, i=1, \ldots, d$. We are going to prove that

$$
\iint_{\mathbf{R}^{+} \times \mathbf{R}^{d}} \operatorname{sgn}_{+}\left(u_{\varepsilon}-\lambda\right) \sum_{i=1}^{d}\left(\left(f_{i}\left(u_{\varepsilon}\right)-f_{i}(\lambda)\right)-c_{i}\left(u_{\varepsilon}-\lambda\right)\right) \frac{\partial\left(B_{1} \varphi\right)}{\partial x_{i}} d x d t \geq o(1),
$$

for $U_{L}-U_{R}>0$ small enough, which, combined with (3.15), will prove that the function $u$ is an entropy solution to (1.2), (1.3). 
Arguing in the completely same way as in the proof of Theorem 3.1, we reach to the following estimate:

$$
\begin{aligned}
& \iint_{\mathbf{R}^{+} \times \mathbf{R}^{d}} \operatorname{sgn}_{+}\left(u_{\varepsilon}-\lambda\right) \sum_{i=1}^{d}\left(\left(f_{i}\left(u_{\varepsilon}\right)-f_{i}(\lambda)\right)-c_{i}\left(u_{\varepsilon}-\lambda\right)\right) \frac{\partial\left(B_{1} \varphi\right)}{\partial x_{i}} d x d t \\
& =\int_{\mathbf{R}^{+}} \int_{\Omega_{L}^{t}}\left[\operatorname{sgn}_{+}\left(U_{L}-\lambda\right) \sum_{i=1}^{d}\left(\left(f_{i}\left(U_{L}\right)-f_{i}(\lambda)\right)-c_{i}\left(U_{L}-\lambda\right)\right)\right. \\
& \left.\quad-\operatorname{sgn}_{+}\left(U_{R}-\lambda\right) \sum_{i=1}^{d}\left(\left(f_{i}\left(U_{R}\right)-f_{i}(\lambda)\right)-c_{i}\left(U_{R}-\lambda\right)\right)\right] \frac{\partial\left(B_{1} \varphi\right)}{\partial x_{i}} d x d t+o(1)
\end{aligned}
$$

Next, notice that if $\lambda>U_{L}>U_{R}$ or $U_{L}>U_{R}>\lambda$ (recall that $U_{L}>U_{R}$; see (1.10), then the subintegral expression on the right-hand side of (3.17) is equal to zero, which means that (3.16) is fulfilled with the equality sign. So, assume that $U_{R}<\lambda<U_{L}$. Relation (3.17) reduces to

$$
\begin{aligned}
& \iint_{\mathbf{R}^{+} \times \mathbf{R}^{d}} \operatorname{sgn}_{+}\left(u_{\varepsilon}-\lambda\right) \sum_{i=1}^{d}\left(\left(f_{i}\left(u_{\varepsilon}\right)-f_{i}(\lambda)\right)-c_{i}\left(u_{\varepsilon}-\lambda\right)\right) \frac{\partial\left(B_{1} \varphi\right)}{\partial x_{i}} d x d t \\
& =\int_{\mathbf{R}^{+}} \int_{\Omega_{L}^{t}}\left[\sum_{i=1}^{d}\left(\left(f_{i}\left(U_{L}\right)-f_{i}(\lambda)\right)-c_{i}\left(U_{L}-\lambda\right)\right)\right] \frac{\partial\left(B_{1} \varphi\right)}{\partial x_{i}} d x d t+o(1) .
\end{aligned}
$$

Applying the Gauss-Ostrogradskii formula, we conclude that

$$
\begin{aligned}
& \int_{\mathbf{R}^{+}} \int_{\Omega_{L}^{t}}\left[\sum_{i=1}^{d}\left(\left(f_{i}\left(U_{L}\right)-f_{i}(\lambda)\right)-c_{i}\left(U_{L}-\lambda\right)\right)\right] \frac{\partial\left(B_{1} \varphi\right)}{\partial x_{i}} d x d t \\
& =\left(U_{L}-\lambda\right) \int_{\mathbf{R}^{+}} \int_{\Gamma_{L}^{t}}\left\langle\vec{n}_{t}, F\left(U_{L}, \lambda\right)\right\rangle B_{1} \varphi d s^{\prime} d t
\end{aligned}
$$

where $\Gamma_{L}^{t}$ is the boundary of the set $\Omega_{L}^{t}, \vec{n}_{t}$ is the unit outer normal on $\Gamma_{L}^{t}$, and

$$
\begin{aligned}
& F\left(U_{L}, \lambda\right) \\
& \quad=\left(\frac{f_{1}\left(U_{L}\right)-f_{1}(\lambda)}{U_{L}-\lambda}-\frac{f_{1}\left(U_{L}\right)-f_{1}\left(U_{R}\right)}{U_{L}-U_{R}}, \ldots, \frac{f_{d}\left(U_{L}\right)-f_{d}(\lambda)}{U_{L}-\lambda}-\frac{f_{d}\left(U_{L}\right)-f_{d}\left(U_{R}\right)}{U_{L}-U_{R}}\right) .
\end{aligned}
$$

Next, notice that $\vec{n}_{t}$ is actually normal on $\Gamma_{L}^{0}=\Gamma_{L}$ translated along the new characteristics at the level $t$. Since the set $\Gamma_{L}^{0}$ is given by $\Gamma_{L}^{0}=\left\{x_{0} \in \mathbf{R}^{d}: u_{1}\left(x_{0}\right)=U_{L}\right\}$, we conclude that the normal on $\Gamma_{L}^{0}$ is given by $\vec{n}_{0}=\nabla u_{1}\left(x_{0}\right)$ (assume for simplicity that $\left.\left\|\nabla u_{1}\left(x_{0}\right)\right\|=1\right)$. Thus, $\vec{n}_{t}=\left.\nabla_{x_{0}} u_{1}\left(x_{0}\right)\right|_{x_{0}=x_{0}(t, x, \varepsilon)}, x \in \Gamma_{L}^{t}$, where $x_{0}(t, x, \varepsilon)$ is the inverse function of the new characteristics $X$ given by (2.7) (the existence of the inverse 
function is proved in Lemma 2.4). Substituting such $\vec{n}_{t}$ into (3.19), we obtain

$$
\begin{array}{r}
\int_{\mathbf{R}^{+}} \int_{\Omega_{L}^{t}}\left[\sum_{i=1}^{d}\left(\left(f_{i}\left(U_{L}\right)-f_{i}(\lambda)\right)-c_{i}\left(U_{L}-\lambda\right)\right)\right] \frac{\partial\left(B_{1} \varphi\right)}{\partial x_{i}} d x d t \\
=\left(U_{L}-\lambda\right) \int_{\mathbf{R}^{+}} \int_{\Gamma_{L}^{t}} \sum_{i=1}^{d}\left(\frac{f_{i}\left(U_{L}\right)-f_{i}(\lambda)}{U_{L}-\lambda}-\frac{f_{i}\left(U_{L}\right)-f_{i}\left(U_{R}\right)}{U_{L}-U_{R}}\right) \\
\times\left.\frac{\partial u_{1}}{\partial x_{0 i}}\right|_{x_{0}=x_{0}\left(t, x\left(s^{\prime}\right), \varepsilon\right)} B_{1} \varphi d s^{\prime} d t
\end{array}
$$

Using Taylor's formula with the integral remainder term, we have for any $i \in\{1, \ldots, d\}$ :

$$
\begin{gathered}
\frac{f_{i}\left(U_{L}\right)-f_{i}(\lambda)}{U_{L}-\lambda}=-f_{i}^{\prime}\left(U_{L}\right)+\frac{1}{2}\left(U_{L}-\lambda\right) f_{i}^{\prime \prime}\left(U_{L}\right)+\frac{1}{2} \frac{1}{U_{L}-\lambda} \int_{U_{L}}^{\lambda}(\lambda-t)^{2} f_{i}^{\prime \prime \prime}(t) d t, \\
\frac{f_{i}\left(U_{L}\right)-f_{i}\left(U_{R}\right)}{U_{L}-U_{R}}=-f_{i}^{\prime}\left(U_{L}\right)+\frac{1}{2}\left(U_{L}-U_{R}\right) f_{i}^{\prime \prime}\left(U_{L}\right)+\frac{1}{2} \frac{1}{U_{L}-U_{R}} \int_{U_{L}}^{U_{R}}\left(U_{R}-t\right)^{2} f_{i}^{\prime \prime \prime}(t) d t .
\end{gathered}
$$

Subtracting the latter terms and noticing that

$$
\begin{aligned}
& \frac{1}{2} \frac{1}{U_{L}-\lambda} \int_{U_{L}}^{\lambda}(\lambda-t)^{2} f_{i}^{\prime \prime \prime}(t) d t-\frac{1}{2} \frac{1}{U_{L}-U_{R}} \int_{U_{L}}^{U_{R}}\left(U_{R}-t\right)^{2} f_{i}^{\prime \prime \prime}(t) d t \\
& =\mathcal{O}\left(\left|U_{R}-\lambda\right|^{2}+\left|\left(U_{R}-\lambda\right)\left(U_{L}-\lambda\right)\right|\right),
\end{aligned}
$$

we get

$$
\begin{aligned}
& \sum_{i=1}^{d}\left(\frac{f_{i}\left(U_{L}\right)-f_{i}(\lambda)}{U_{L}-\lambda}-\frac{f_{i}\left(U_{L}\right)-f_{i}\left(U_{R}\right)}{U_{L}-U_{R}}\right) \\
& =\left(\sum_{i=1}^{d} f_{i}^{\prime \prime}\left(U_{L}\right) \frac{U_{R}-\lambda}{2}+\mathcal{O}\left(\left|U_{R}-\lambda\right|^{2}+\left|\left(U_{R}-\lambda\right)\left(U_{L}-\lambda\right)\right|\right)\right)
\end{aligned}
$$


From here and (3.20), we conclude that

$$
\begin{aligned}
& \int_{\mathbf{R}^{+}} \int_{\Omega_{L}^{t}}\left[\sum_{i=1}^{d}\left(\left(f_{i}\left(U_{L}\right)-f_{i}(\lambda)\right)-c_{i}\left(U_{L}-\lambda\right)\right)\right] \frac{\partial\left(B_{1} \varphi\right)}{\partial x_{i}} d x d t \\
& =-\left.\left(U_{L}-\lambda\right)\left(\lambda-U_{R}\right) \int_{\mathbf{R}^{+}} \int_{\Gamma_{L}^{t}} \sum_{i=1}^{d} f^{\prime \prime}\left(U_{L}\right) \frac{\partial u_{1}}{\partial x_{0 i}}\right|_{x_{0}=x_{0}\left(t, x\left(s^{\prime}\right), \varepsilon\right)} B_{1} \varphi d s^{\prime} d t \\
& +\int_{\mathbf{R}^{+}} \int_{\Gamma_{L}^{t}} \mathcal{O}\left(\left|\left(U_{L}-\lambda\right)\left(U_{R}-\lambda\right)^{2}\right|+\left|\left(U_{R}-\lambda\right)\left(U_{L}-\lambda\right)^{2}\right|\right) B_{1} \varphi d s^{\prime} d t \\
& =-\left.\left(U_{L}-\lambda\right)\left(\lambda-U_{R}\right) \int_{\mathbf{R}^{+}} \int_{\Gamma_{L}^{t}}\left(\sum_{i=1}^{d} f^{\prime \prime}\left(u_{1}\right) \frac{\partial u_{1}}{\partial x_{0 i}}\right)\right|_{x_{0}=x_{0}\left(t, x\left(s^{\prime}\right), \varepsilon\right)} B_{1} \varphi d s^{\prime} d t \\
& +\int_{\mathbf{R}^{+}} \int_{\Gamma_{L}^{t}} \mathcal{O}\left(\left|\left(U_{L}-\lambda\right)\left(U_{R}-\lambda\right)^{2}\right|+\left|\left(U_{R}-\lambda\right)\left(U_{L}-\lambda\right)^{2}\right|\right) B_{1} \varphi d s^{\prime} d t \\
& =\int_{\mathbf{R}^{+}} \int_{\Gamma_{L}^{t}}\left(K(s)\left(U_{L}-\lambda\right)\left(\lambda-U_{R}\right)\right.
\end{aligned}
$$

for $U_{L}-U_{R}$ small enough. Above, we used $u_{1}\left(x_{0}(t, x, \varepsilon)\right)=U_{L}$ for $x \in \Gamma_{L}^{t}$ (the second equality) and (1.6) (the third equality).

Together with (3.18), this proves (3.16) and concludes the theorem.

Acknowledgement. We would like to thank the referee whose excellent comments and remarks helped us to improve the paper substantially.

\section{REFERENCES}

[1] I. Berre, H. K. Dahle, K. H. Karlsen, K.-A. Lie, J. R. Natvig, Time-of-Flight + Fast Marching + Transport Collapse: An Alternative to Streamlines for Two-Phase Porous Media Flow with Capillary Forces?, In Computational Methods in Water Resources (Delft, The Netherlands, 2002), 995-1002, Elsevier, 2002.

[2] C. M. Dafermos, Hyperbolic Conservation Laws in Continuum Physics, Springer-Verlag, BerlinNew York, 2000. MR1763936 (2001m:35212)

[3] V. G. Danilov, Generalized Solution Describing Singularity Interaction, International J. of Mathematics and Mathematical Sciences 29 (2002), 481-494. MR1896252 (2003m:35149)

[4] V. G. Danilov, Weak asymptotic solution of phase-field system in the case of confluence of free boundaries in the Stefan problem with underheating, European J. of Applied Mathematics 18 (2007), 537-570. MR2360622 (2009a:35272)

[5] V. G. Danilov, G. A. Omelianov, Weak asymptotic method for the study of propagation and interaction of infinitely narrow $\delta$ solitons, Electronic J. of Differential Equations, 2003 (2003), $1-27$.

[6] V.G. Danilov, V. M. Shelkovich, Dynamics of propagation and interaction of $\delta$-shock waves in conservation law systems, J. Differential Equations 211 (2005), 333-381. MR 2125546 (2006f:35173)

[7] V. G. Danilov, D. Mitrović, Weak asymptotics of shock wave formation process, Nonlinear Analysis: Theory, Methods and Applications, 61 (2005), 613-635. MR2126617 (2006a:35193)

[8] V. G. Danilov, D. Mitrović, Delta shock wave formation in the case of triangular hyperbolic system of conservation laws, J. Differential Equations 245 (2008), 3704-3734. MR2462701 (2009h:35264)

[9] V. G. Danilov, D. Mitrović, Smooth approximations of global in time solutions to scalar conservation laws, Abstract and Applied Analysis, 2009 (2009), 1-26. MR2485639 (2010d:35224) 
[10] V. G. Danilov, G. A. Omel'yanov, and V. M. Shelkovich, Weak asymptotics method and interaction of nonlinear waves. In: Asymptotic Methods for Wave and Quantum Problems, M. V. Karasev, ed., AMS Transl., Ser. 2, Vol. 208, 33-164. MR1995392 (2004f:35021)

[11] V. G. Danilov, V. M. Shelkovich, Delta-shock wave type solution of hyperbolic systems of conservation laws, Quart. Appl. Math. 63 (2005), 401-427. MR2169026 (2006j:35158)

[12] R. Flores-Espinosa, G. A. Omel'yanov, Weak asymptotics for the problem of interaction of two shock waves, Nonlinear Phenomena in Complex Systems, 8 (2005), 331-341. MR2240498 (2007c:35108)

[13] R. Flores-Espinosa, G. A. Omel'yanov, Asymptotic behavior for the centered-rarefaction appearance problem. Electronic J. Differential Equations, 2005 (2005), 1-25. MR2195544 (2006j:35160)

[14] M. G. Garcia, G. A. Omel'yanov, Kink-antikink interaction for semilinear wave equation with a small parameter, Electronic J. of Differential Equations, 2009 (2009), 1-26. MR2495850 (2010d:35233)

[15] M. G. Garcia, R. Flores-Espinosa, G. A. Omel'yanov, Interaction of shock waves in gas dynamics. Uniform in time asymptotics, International J. Mathematics and Mathematical Sciences, 19 (2005), 3111-3126. MR2206088 (2006i:35235)

[16] J. Glimm, D. Marchesin, O. McBryan, Unstable fingers in two phase flows, Commun. Pure. Appl. Math., 34 (1981), 53-75. MR600572 (82e:76069)

[17] A. M. Il'in, Matching of Asymptotic Expansions of Solutions of Boundary Value Problem, Nauka, Moscow, 1989, English transl., American Mathematical Society, Providence, RI, 1992. MR1182791 (93g:35016)

[18] A. M. Il'in, S. V. Zakharov, From a weak discontinuity to the gradient catastrophe, Mat. Sb. 193 (2001), 3-18. MR.1867014 (2002k:35198)

[19] G. Kossioris, I. Shyuichi, Geometric singularities for solutions of single conservation law, Arch. Rational Mech. Anal., 139 (1997), 255-290. MR 1480242 (98j:35116)

[20] S. N. Kruzhkov, First order quasilinear equations in several independent variables, Math. USSR Sb. 10 (1970), 217-243.

[21] D. A. Kulagin, G. A. Omel'yanov, Interaction of kinks for semilinear wave equations with a small parameter, Nonlinear Analysis: Theory, Methods and Applications, 65 (2006), 347-378. MR:2228433 (2007f:35197)

[22] A. M. Il'in, Matching of Asymptotic Expansions of Solutions of Boundary Value Problems, Nauka, Moscow, 1989, English transl., American Mathematical Society, Providence, RI, 1992. MR1182791 (93g:35016)

[23] A. M. Il'in, S. V. Zakharov, From a weak discontinuity to the gradient catastrophe, Mat. Sb. 193 (2001), 3-18. MR1867014 (2002k:35198)

[24] D. Mitrovic, V. Bojkovic, V. Danilov, Linearization of the Riemann problem for a triangular system of conservation laws and delta shock wave formation process, Mathematical Methods in the Applied Sciences, 33 (2010), 904-921. MR2662315 (2011d:35310)

[25] D. Mitrovic, J. Susic, Global in time solution to Hopf equation and application to a non-strictly hyperbolic system of conservation laws, Electronic J. of Differential Equations, 2007 (2007), 1-22. MR2349942(2008f:35241)

[26] S. Nakane, Formation of shocks for a single conservation law, SIAM Journal of Math. Anal., 19 (1988), 1391-1408. MR.965259 (89k:35142)

[27] E. Yu. Panov, V. M. Shelkovich, $\delta^{\prime}$-Shock waves as a new type of solutions to systems of conservation laws, J. of Differential Equations 228 (2006), 49-86. MR.2254184 (2007f:35188)

[28] D. Wagner, The Riemann problem in two space dimensions for a single conservation law, SIAM J. Math. Anal., 14 (1983), 534-559. MR697528(84f:35092) 